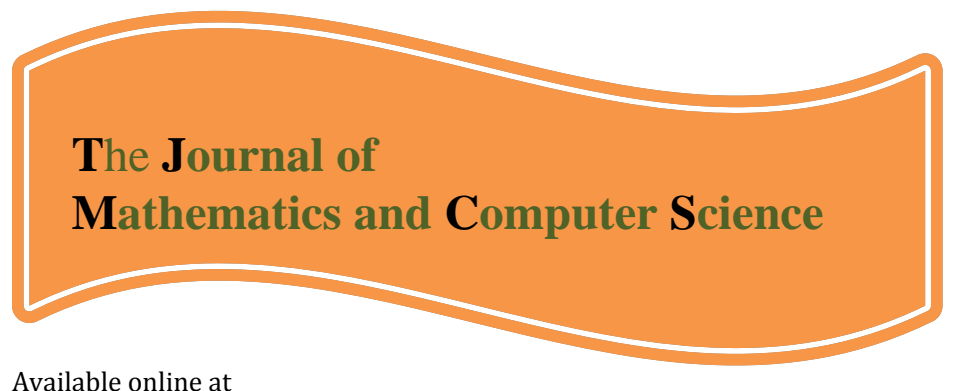

Available online at

http://www.TJMCS.com

The Journal of Mathematics and Computer Science Vol .1 No.4 (2010) 377-385

\title{
Application of Homotopy perturbation method for fuzzy integral equations
}

\author{
Mashallah Matinfar1,*, Mohammad Saeidy² \\ m.matinfar@umz.ac.ir \\ m.saidy@umz.ac.ir
}

Received: July 2010, Revised: October 2010

Online Publication: December 2010

\begin{abstract}
In this paper, an application of homotopy perturbation method (HPM) is applied to solve linear fuzzy Fredholm integral equation. Comparison are made between the exact solution and solution of homotopy perturbation method. The results reveal that the homotopy analysis method is very effective and simple.
\end{abstract}

Keywords: Fuzzy number; Fredholm integral equation; Homotopy perturbation method.

\section{Introduction}

The homotopy perturbation method[5], proposed first by He in 1998 and was further developed and improved by He[6-9]. The method yields a very rapid convergence of the solution series in the most cases. Usually, one iteration leads to high accuracy of the solution. Although goal of He's homotopy perturbation method was to find a technique to unify linear and nonlinear, ordinary or partial differential equations for solving initial and boundary value problems. The topics of fuzzy integral equations (FIE) which attracted growing interest for some time, particularly in relation to fuzzy control, have been developed in recent years. In this paper, we apply He's homotopy perturbation method to linear Fredholm fuzzy integral equations of the second kind. The results reveal that the proposed method is very effective and simple.

\footnotetext{
$1,{ }^{*}$ Corresponding author: Department of mathematics-University of Mazandaran-Iran

2 Department of mathematics-University of Mazandaran-Iran
} 


\section{Preliminaries}

In this section the most basic notations used in fuzzy calculus are introduced. We start with defining a fuzzy number.

Dedfinition 1. [1] A fuzzy number is a map $u: R \rightarrow I=[0 ; 1]$ which satisfies

i. $u$ is upper semi-continuous.

ii. $u(\mathrm{x})=0$ outside some interval [c, d] .

iii. There exist real numbers $\mathrm{a}, \mathrm{b}$ such that $\mathrm{c} \leq \mathrm{a} \leq \mathrm{b} \leq \mathrm{d}$, where

1. $u(\mathrm{x})$ is monotonic increasing on $[\mathrm{c}, \mathrm{a}]$,

2. $u(\mathrm{x})$ is monotonic decreasing on $[\mathrm{b}, \mathrm{d}]$,

3. $u(\mathrm{x})=1, \mathrm{a} \leq \mathrm{x} \leq \mathrm{b}$.

The set of all such fuzzy numbers is represented by $E^{1}$. An equivalent parametric definition of fuzzy numbers is given in [3] as

Definition 2. An arbitrary fuzzy number in parametric form is represented by an ordered pair of functions $(\underline{u}(r), \bar{u}(r)), 0 \leq r \leq 1$, which satisfying the following requirements:

i. $\underline{u}(r)$ is a bounded left-continuous non-decreasing function over $[0,1]$.

ii. $\bar{u}(r)$ is a bounded left-continuous non-increasing function over $[0,1]$.

iii. $\underline{u}(r) \leq \bar{u}(r), 0 \leq r \leq 1$.

For arbitrary $u=(\bar{u}, \underline{u}), v=(\bar{v}, \underline{v})$ and $\mathrm{k}>0$ we define addition $(u+v)$ and multiplication by $\mathrm{k}$ as

$(\overline{u+v)}(r)=\underline{u}(r)+\underline{v}(r)$,
$\overline{(\overline{u+v})}(r)=\bar{u}(r)+\bar{v}(r)$,

$(\underline{k u})(r)=k \underline{u}(r), \quad(\overline{k u})(r)=k \bar{u}(r)$,

The collection of all the fuzzy numbers with addition and multiplication as de_ned by Eqs. (1) and (2) is denoted by $E^{1}$ and is a convex cone. It can be shown that Eqs. (1) and (2) are equivalent to the addition and multiplication as defined by using the $\alpha$-cut approach[4] and the extension principles[8]. We will next de_ne the fuzzy function notation and a metric D in $E^{1}$ [4].

Definition 3. For arbitrary fuzzy numbers $u=(\bar{u}, \underline{u}), v=(\bar{v}, \underline{v})$ the quantity

$D(u, v)=\max \left\{\sup _{0 \leq r \leq 1}|\underline{u}(r)-\underline{v}(r)|, \sup _{0 \leq r \leq 1}|\bar{u}(r)-\bar{v}(r)|\right\}$

is the distance between $u$ and $v$. This metric is equivalent to the one used by Puri and Ralescu[11] and Kaleva[12]. It is shown[14] that $\left(E^{1}, \mathrm{D}\right)$ is a complete metric space. We now follow Goetschel and Voxman[13] and define the integral of a fuzzy function using the Riemann integral concept. 
Let $f:[a, b] \rightarrow E^{1}$. For each partition $p=\left\{t_{0}, t_{1}, \ldots, t_{n}\right\}$ of $[a, b]$ with $h=\max \left|t_{i}-t_{i-1}\right|$ and for arbitrary $\xi_{i}: t_{i-1} \leq \xi_{i} \leq t_{i}, 1 \leq i \leq n$ let

$$
R_{p}=\sum_{i=1}^{n} f\left(\xi_{i}\right)\left(t_{i}-t_{i-1}\right)
$$

The definite integral of $f(t)$ over $[a, b]$ is

$$
\int_{a}^{b} f(t) d t=\lim R_{p}, h \rightarrow 0
$$

provided that this limit exists in the metric $D$.

If the fuzzy function $f(t)$ is continuous in the metric $D$, its de_nite integral exists[4].

Furthermore,

$$
\left(\int_{\underline{a}}^{b} f(t, r) d t\right)=\int_{a}^{b} \underline{f}(t, r) d t, \quad\left(\overline{\int_{a}^{b} f(t, r) d t}\right)=\int_{a}^{b} \bar{f}(t, r) d t .
$$

More details about the properties of the fuzzy integral are given in[4,12].

\section{Fuzzy integral equation}

The integral equations which are discussed in this section are the Fredholm equations of the second kind. The Fredholm integral equation of the second kind is[2]

$$
F(t)=f(t)+\beta \int_{a}^{b} K(s, t) F(s) d s
$$

where $\beta \succ 0, K(s, t)$ is an arbitrary given kernel function over the square $a \leq s, b \leq t$ and $f(t)$ is a given function of $t: a \leq t \leq b$. If $f(t)$ is a crisp function then the solutions of Eq. (5) is crisp as well. However, if $f(t)$ is a fuzzy function, this equations may only possess fuzzy solution. Sufficient conditions for the existence of a unique solution to the fuzzy Fredholm integral equation of the second kind, i.e. to Eq. (5) where $f(t)$ is a fuzzy function, are given in[13].

Now, we introduce parametric form of a FFIE-2 with respect to Definition 2. Let $(\underline{f}(t, r), \bar{f}(t, r))$ and $(\underline{u}(t, r), \bar{u}(t, r)), 0 \leq r \leq 1$ and $t \in[a, b]$ are parametric form of $f(t)$ and $u(t)$, respectively then, parametric form of FFIE-2 is as follows:

$$
\begin{aligned}
& \underline{u}(t, r)=\underline{f}(t, r)+\beta \int_{a}^{b} v_{1}(s, t, \underline{u}(s, r), \bar{u}(s, r)) d s, \\
& \bar{u}(t, r)=\bar{f}(t, r)+\beta \int_{a}^{b} v_{2}(s, t, \underline{u}(s, r), \bar{u}(s, r)) d s,
\end{aligned}
$$

where

$$
v_{1}(s, t, \underline{u}(s, r), \bar{u}(s, r))=\left\{\begin{array}{lc}
K(s, r) \underline{u}(s, r), & K(s, r) \geq 0, \\
K(s, r) \bar{u}(s, r), & K(s, r) \leq 0
\end{array}\right.
$$

and 


$$
v_{2}(s, t, \underline{u}(s, r), \bar{u}(s, r))= \begin{cases}K(s, r) \bar{u}(s, r), & K(s, r) \geq 0, \\ K(s, r) \underline{u}(s, r), & K(s, r) \leq 0,\end{cases}
$$

for each $0 \leq r \leq 1$ and $t \in[a, b]$. We can see that (6) is a system of linear Fredholm integral equations in crisp case for each $0 \leq r \leq 1$ and $t \in[a, b]$. In next section, we explain homotopy analysis method as a numerical algorithm for approximating solution of this system of linear integral equations in crisp case then, we find approximate solutions for $\underline{u}(t, r)$ and $\bar{u}(t, r)$ for each $0 \leq r \leq 1$ and $t \in[a, b]$.

\section{Basic idea of homotopy perturbation method}

To illustrate the basic ideas of this method, we consider the following nonlinear differential equation

$$
A(U)-f(r)=0, \quad r \in \Omega,
$$

with the following boundary conditions

$$
B\left(u, \frac{\partial u}{\partial r}\right)=0, \quad r \in \Gamma,
$$

Where $A$ is a general differential operator, $B$ a boundary operator, $f(r)$ is a known analytical function and $\Gamma$ is the boundary of the domain $\Omega$. The operator $A$ can be decomposed into two operators, $L$ and $N$, where $L$ is a linear, and $N$ a nonlinear operator. Therefore (7) can be written as follows:

$$
L(u)+N(u)-f(r)=0 .
$$

Using the homotopy technique, we construct a homotopy $U(r, p): \Omega \times[0,1] \rightarrow R$, which satisfies:

$$
H(U, p)=(1-p)\left[L(U)-L\left(u_{0}\right)\right]+p[A(U)-f(r)]=0, \quad p \in[0,1], r \in \Omega .
$$

or

$$
H(U, p)=L(U)-L\left(u_{0}\right)+p L\left(u_{0}\right)+P[N(U)-f(r)]=0,
$$

where $p \in[0,1]$ is an embedding parameter, $u_{0}$ is an initial approximation for the solution of (7), which satisfies the boundary conditions. Obviously, from (10) and(11) we will have

$$
H(U, 0)=L(U)-L\left(u_{0}\right), \quad H(U, 1)=A(U)-f(r) .
$$

The changing process of $\mathrm{p}$ form zero to unity is just that of $U(r, p)$ from $u_{0}(r)$ to $u(r)$. In topology, this is called homotopy. According to the (HPM), we can first use the embedding parameter $p$ as a small parameter, and assume that the solution of (10) and (11) can be written as a power series in $p$ :

$$
U=U_{0}+p U_{1}+p^{2} U_{2}+p^{3} U_{3}+\ldots
$$

Setting $p=1$, results in the approximate solution of (7)

$$
u=\lim _{p \rightarrow 1} U=U_{0}+U_{1}+U_{2}+\cdots
$$

The combination of the perturbation method and the homotopy method is called the homotopy perturbation method (HPM), which has eliminated the limitations of the traditional perturbation methods. On the other hand, this technique can have full advantage of the traditional perturbation techniques. The series (14) is convergent for most cases. Some criteria is suggested for convergence of the series (14), in [5].

\section{Application}

In this section, we apply this algorithm to two examples. 
Example 1. Consider the fuzzy Fredholm integral equation with[1]

$$
\begin{aligned}
& \underline{f}(t, r)=\sin \left(\frac{t}{2}\right)\left(\frac{13}{15}\left(r^{2}+r\right)+\frac{2}{15}\left(4-r^{3}-r\right)\right) \\
& \bar{f}(t, r)=\sin \left(\frac{t}{2}\right)\left(\frac{2}{15}\left(r^{2}+r\right)+\frac{13}{15}\left(4-r^{3}-r\right)\right)
\end{aligned}
$$

and kernel

$$
K(s, t)=0.1 \sin (s) \sin \left(\frac{t}{2}\right), \quad 0 \leq s, t \leq 2 \pi, \text { and } a=0, b=2 .
$$

The exact solution in this case is given by

$$
\underline{u}(t, r)=\sin \left(\frac{t}{2}\right)\left(r^{2}+r\right), \quad \bar{u}(t, r)=\sin \left(\frac{t}{2}\right)\left(4-r^{3}-r\right),
$$

By homotopy perturbation method, we construct the following homotopies:

$$
(1-p)\left(\underline{v}(r, t)-\underline{u}_{0}(t, r)\right)+p\left\{\underline{u}(t, r)-\underline{f}(t, r)-\int_{0}^{\pi} k(s, t) \underline{v}(s, r) d s-\int_{\pi}^{2 \pi} k(s, t) \bar{v}(s, r) d s\right\}=0,
$$

or

$$
\underline{v}(t, r)-\underline{u}_{0}(t, r)=p\left\{\int_{0}^{\pi} k(s, t) \underline{v}(s, r) d s+\int_{\pi}^{2 \pi} k(s, t) \bar{v}(s, r) d s\right\}
$$

and

$$
(1-p)\left(\bar{v}(r, t)-\bar{u}_{0}(t, r)\right)+p\left\{\bar{u}(t, r)-\bar{f}(t, r)-\int_{0}^{\pi} k(s, t) \bar{v}(s, r) d s-\int_{\pi}^{2 \pi} k(s, t) \underline{v}(s, r) d s\right\}=0,
$$

or

$$
\bar{v}(t, r)-\bar{u}_{0}(t, r)=p\left\{\int_{0}^{\pi} k(s, t) \bar{v}(s, r) d s+\int_{\pi}^{2 \pi} k(s, t) \underline{v}(s, r) d s\right\}
$$

Suppose the solution of Example(5.1) to be in the following forms

$$
\underline{v}=\underline{v}_{0}+p \underline{v}_{1}+p^{2} \underline{v}_{2}+\cdots, \quad \bar{v}=\bar{v}_{0}+p \bar{v}_{1}+p^{2} \bar{v}_{2}+\cdots,
$$

Substituting (18) into (16) and (17), and equating the coefficients of the terms with the identical powers of $p$,

$$
\begin{aligned}
p^{0}: \underline{v}_{0} & =\underline{u}_{0}, \\
\vdots & \\
p^{j}: \underline{v}_{j} & =\int_{0}^{\pi} k(s, t) \underline{v}_{j-1}(s, r) d s+\int_{\pi}^{2 \pi} k(s, t) \bar{v}_{j-1}(s, r) d s, \quad j=1,2, \ldots,
\end{aligned}
$$

and 


$$
\begin{aligned}
p^{0}: \bar{v}_{0} & =\overline{u_{0}} \\
& \vdots \\
p^{j}: \bar{v}_{j} & =\int_{0}^{\pi} k(s, t) \bar{v}_{j-1}(s, r) d s+\int_{\pi}^{2 \pi} k(s, t) \underline{v}_{j-1}(s, r) d s, \quad j=1,2, \ldots,
\end{aligned}
$$

Starting with $\underline{v}_{0}=\underline{u}_{0}=\frac{1}{15} \sin \left(\frac{t}{2}\right)\left(13 r^{2}+11 r+8-2 r^{3}\right)$ and $\bar{v}_{0}=\bar{u}_{0}=\frac{1}{15} \sin \left(\frac{t}{2}\right)\left(2 r^{2}-11 r+52-13 r^{3}\right)$ also by using (19) and (20), we obtain

$$
\begin{gathered}
\underline{v}_{1}(t, r)=\frac{22}{225} \sin \left(\frac{t}{2}\right)\left(r^{2}+2 r-4+r^{3}\right), \\
\underline{v}_{2}(t, r)=\frac{88}{3375} \sin \left(\frac{t}{2}\right)\left(r^{2}+2 r-4+r^{3}\right), \\
\vdots
\end{gathered}
$$

and

$$
\begin{aligned}
& \underline{v}_{1}(t, r)=-\frac{22}{225} \sin \left(\frac{t}{2}\right)\left(r^{2}+2 r-4+r^{3}\right), \\
& \underline{v}_{2}(t, r)=-\frac{88}{3375} \sin \left(\frac{t}{2}\right)\left(r^{2}+2 r-4+r^{3}\right),
\end{aligned}
$$

The approximate solution of Example (5.1) can be obtained by setting $p=1$,

$$
\underline{u}=\underline{v}_{0}+\underline{v}_{1}+\underline{v}_{2}+\underline{v}_{3}=\frac{1}{50625}\left(50479 r^{2}-128 r^{3}+50369 r+512\right) \sin \left(\frac{t}{2}\right),
$$

and

$$
\bar{u}=\bar{v}_{0}+\bar{v}_{1}+\bar{v}_{2}+\bar{v}_{3}=-\frac{1}{50625}\left(50479 r^{2}-128 r^{3}+50369 r+512\right) \sin \left(\frac{t}{2}\right),
$$

Example 2. Consider the following fuzzy Fredholm integral equation with [1]

$$
\begin{aligned}
& \underline{f}(t, r)=r t+\frac{3}{26}-\frac{3}{26} r-\frac{1}{13} t^{2}-\frac{1}{13} t^{2} r \\
& \bar{f}(t, r)=2 t-r t+\frac{3}{26} r+\frac{1}{13} t^{2} r-\frac{3}{26}-\frac{3}{13} t^{2},
\end{aligned}
$$

and kernel

$$
K(s, t)=\frac{s^{2}+t^{2}-2}{13}, \quad 0 \leq s, t \leq 2 \quad \text { and } a=0, b=2 .
$$

The exact solution in this case is given by

$$
\underline{u}(t, r)=r t, \quad \bar{u}(t, r)=(2-r) t,
$$

By homotopy perturbation method, we construct the following homotopies: 


$$
(1-p)\left(\underline{v}(r, t)-\underline{u}_{0}(t, r)\right)+p\left\{\underline{u}(t, r)-\underline{f}(t, r)-\int_{0}^{1} k(s, t) \bar{v}(s, r) d s-\int_{1}^{2} k(s, t) \underline{v}(s, r) d s\right\}=0,
$$

or

$$
\underline{v}(t, r)-\underline{u}_{0}(t, r)=p\left\{\int_{0}^{1} k(s, t) \underline{v}(s, r) d s+\int_{1}^{2} k(s, t) \bar{v}(s, r) d s\right\}
$$

and

$$
(1-p)\left(\bar{v}(r, t)-\bar{u}_{0}(t, r)\right)+p\left\{\bar{u}(t, r)-\bar{f}(t, r)-\int_{0}^{1} k(s, t) \underline{v}(s, r) d s-\int_{1}^{2} k(s, t) \bar{v}(s, r) d s\right\}=0,
$$

or

$$
\bar{v}(t, r)-\bar{u}_{0}(t, r)=p\left\{\int_{0}^{1} k(s, t) \underline{v}(s, r) d s+\int_{1}^{2} k(s, t) \bar{v}(s, r) d s\right\}
$$

Suppose the solution of Example(5.2) to be in the following forms

$$
\underline{v}=\underline{v}_{0}+p \underline{v}_{1}+p^{2} \underline{v}_{2}+\cdots, \quad \bar{v}=\bar{v}_{0}+p \bar{v}_{1}+p^{2} \bar{v}_{2}+\cdots,
$$

Substituting (124) into (22) and (23), and equating the coefficients of the terms with the identical powers of $p$,

$$
\begin{aligned}
p^{0}: \underline{v}_{0} & =\underline{u}_{0} \\
\vdots & \\
p^{j}: \underline{v}_{j} & =\int_{0}^{1} k(s, t) \bar{v}_{j-1}(s, r) d s+\int_{1}^{2} k(s, t) \underline{v}_{j-1}(s, r) d s, \quad j=1,2, \ldots,
\end{aligned}
$$

and

$$
\begin{aligned}
p^{0}: \bar{v}_{0} & =\bar{u}_{0} \\
& \vdots \\
p^{j}: \bar{v}_{j} & =\int_{0}^{1} k(s, t) \underline{v}_{j-1}(s, r) d s+\int_{1}^{2} k(s, t) \bar{v}_{j-1}(s, r) d s, \quad j=1,2, \ldots,
\end{aligned}
$$

Starting with $\underline{v}_{0}=\underline{u}_{0}=r t+\frac{3}{26}-\frac{3}{26} r-\frac{1}{13} t^{2}-\frac{1}{13} t^{2} r$ and $\bar{v}_{0}=\bar{u}_{0}=2 t-r t+\frac{3}{26} r+\frac{1}{13} t^{2} r-\frac{3}{26}-\frac{3}{13} t^{2}$ also by using (25) and (26), we obtain

$$
\begin{aligned}
& \underline{v}_{1}(t, r)=\frac{29}{338} r-\frac{499}{15070}+\frac{11}{169} t^{2} r+\frac{29}{507} t^{2}, \\
& \underline{v}_{2}(t, r)=\frac{51}{2197} r-\frac{235}{19773}+\frac{22}{2197} t^{2} r+\frac{1298}{98865} t^{2},
\end{aligned}
$$

and

$$
\begin{aligned}
& \underline{v}_{1}(t, r)=-\frac{29}{338} r+\frac{371}{15070}-\frac{11}{169} t^{2} r+\frac{29}{507} t^{2}, \\
& \underline{v}_{2}(t, r)=-\frac{51}{2197} r+\frac{638}{19773}-\frac{22}{2197} t^{2} r+\frac{3278}{98865} t^{2},
\end{aligned}
$$


The approximate solution of Example (4.2) can be obtained by setting $p=1$,

$$
\underline{u}=\sum_{i=0}^{5} \underline{v}_{i}=r t+\frac{2049968}{146614323375}-\frac{16}{371293} r-\frac{242464}{2255604975} t^{2}-\frac{322}{4826809} t^{2} r,
$$

and

$$
\bar{u}=\sum_{i=0}^{5} \bar{v}_{i}=2 t-r t+\frac{16}{371993} r+\frac{32}{4826809} t^{2} r-\frac{10586032}{146614323375}-\frac{3540832}{293228646 \mathrm{~B}} t^{2} .
$$

\subsection{Figurse}

We compare aproximate solution and exact solution in"Fig.1 and Fig. 2".

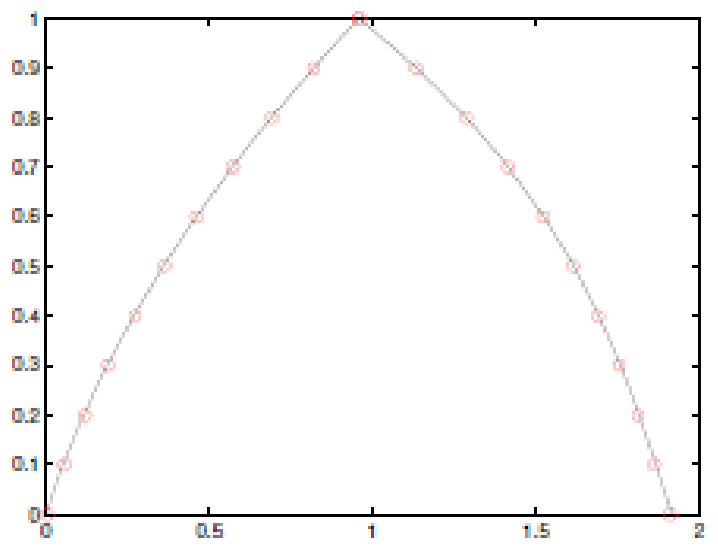

Fig.1.

Open circles: HPM solution; Solid line: Exact solution for Example 4.1(t=1).

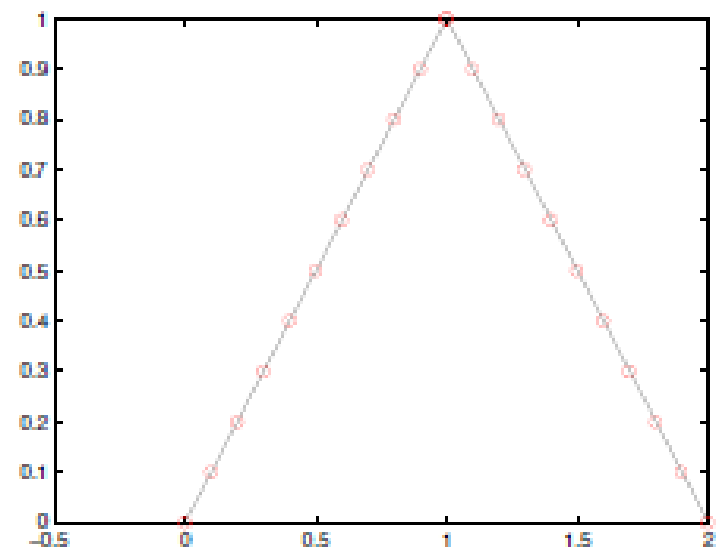

Fig.2.

Open circles: HPM solution; Solid line: Exact solution for Example 4.2(t=1).

\section{Conclusion}

In this paper, the homotopy perturbation method has been successfully applied to find the solution of linear fuzzy Fredholm integral equation of the second kind. It is apparently seen that HPM is a very powerful and efficient technique in finding analytical solutions for wide classes of linear problems. It is 
worth pointing out that this method presents a rapid convergence for the solutions. They also do not require large computer memory and discretization of the variables $t$ and $r$. The computations associated with the examples in this letter were performed using Matlab 7.

\section{References}

[1] Babolian, E., Sadeghi , Goghary, H. and Abbasbandy S., "Numerical solution of linear Fredholm

fuzzy integral equations of the second kind by Adomian method", Appl. Math. Comput., Vol. 161, pp.733-44, 2005.

[9] Buckley, J.J., "Fuzzy eigenvalues and inputoutput analysis", Fuzzy Sets Systems , Vol. 34, pp. 187-195, 1990.

[14] Chang, S.S.L. and Zadeh, L., "On fuzzy mapping and control”, IEEE Trans. Systems Man Cybernet, Vol. 2 , pp. 30-34, 1972.

[13] Goetschel, R. and Voxman, W., "Eigen fuzzy number sets", Fuzzy Sets Systems, Vol. 16, pp. 75-85, 1985.

[3] He, J.H., "Homotopy perturbation technique", Comput. Meth. Appl. Mech. Eng., Vol. 178, pp. 257-262, 1999.

[4] He, J.H., "Approximate analytical solution for seepage flow with fractional derivatives in porous media",Comput. Meth. Appl. Mech. Eng., Vol. 167, N0.1-2, pp. 57-68, 1998.

[5] He, J.H., "Comparison of homotopy perturbtaion method and homotopy analysis Method", Appl. Math. Comput., Vol. 156, pp. 527-539, 2004.

[6] He, J.H., "A coupling method of homotopy technique and perturbation technique for nonlinear problems", Int. J. Nonlinear. Mech. , Vol. 35, No.1, pp. 37-43, 2000.

[7] He, J.H., "Homotopy perturbation method: a new nonlinear analytical technique", Appl. Math. Comput., Vol. 135, pp. 73-79, 2003.

[8] He, J.H., "Some asymptotic methods for strongly nonlinear equations", Int .J. Mod .Phys. B. ,Vol. 20, No. 10, pp. 1141-1199, 2006.

[10] Puri, M.L. and Ralescu, D., "Fuzzy random variables", J. Math. Anal. Appl. , Vol. 114, pp. 409$422,1986$.

[11] Park, J.H., "Intuitionistic fuzzy metric spaces", Chaos, Solitons \& Fractals, Vol. 22, pp. 103946, 2004.

[2] $\mathrm{Wu}, \mathrm{C}$. and Ma, M., "On the integrals, series and integral equations of fuzzy set valued functions", J. Harbin Inst. Technol, Vol. 21, pp. 11-19, 1990.

[12] Zhao, R. and Govind,R., "Solutions of algebraic equations involving generalized fuzzy number”, Inform. Sci., Vol. 56, pp. 199-243, 1991. 